Société d'histoire de la révolution de 1848 et des

révolutions du XIXe siècle

$50 \mid 2015$

Sociétés et forces de sécurité au XIXe siècle

\title{
Grossesses ignorées au prisme de l'infanticide. Savoirs médicaux et décisions de justice au XIXe siècle
}

Ignored Pregnancies and Infanticide. Medical Knowledge and Judicial Decisions

(France $19^{\text {th }}$ century)

Ignorierte Schwangerschaften und Kindesmord. Medizinisches Wissen und gerichtliche Entscheidungen im 19. Jahrhundert

\section{Silvia Chiletti}

\section{(2) OpenEdition}

Journals

Édition électronique

URL : http://journals.openedition.org/rh19/4830

DOI : $10.4000 /$ rh 19.4830

ISSN : $1777-5329$

Éditeur

La Société de 1848

Édition imprimée

Date de publication : 1 juillet 2015

Pagination : 165-179

ISSN : 1265-1354

Référence électronique

Silvia Chiletti, « Grossesses ignorées au prisme de l'infanticide. Savoirs médicaux et décisions de justice au XIXe siècle », Revue d'histoire du XIXe siècle [En ligne], 50 | 2015, mis en ligne le 01 juillet 2015, consulté le 01 mai 2019. URL : http://journals.openedition.org/rh19/4830; DOI : 10.4000/ rh19.4830

Ce document a été généré automatiquement le 1 mai 2019.

Tous droits réservés 


\section{Grossesses ignorées au prisme de l'infanticide. Savoirs médicaux et décisions de justice au XIXe siècle}

Ignored Pregnancies and Infanticide. Medical Knowledge and Judicial Decisions

(France $19^{\text {th }}$ century)

Ignorierte Schwangerschaften und Kindesmord. Medizinisches Wissen und

gerichtliche Entscheidungen im 19. Jahrhundert

Silvia Chiletti

\section{NOTE DE L'AUTEUR}

Cet article est issu d'une recherche post-doctorale menée dans le cadre de la Bourse Fernand Braudel - IFER, COFUND Fondation Maison des sciences de l'homme - Union européenne, réalisée au Centre Alexandre-Koyré - Histoire des sciences et des techniques (UMR 8560). Je remercie les collègues du Centre Alexandre-Koyré et ceux avec qui j'ai eu l'opportunité de discuter de ce travail, notamment Francesca Arena, Emmanuelle Berthiaud, Jacqueline Carroy, Jean-Christophe Coffin, Nicole Edelman et Aude Fauvel.

Est-il possible qu'une femme ignore sa grossesse tout au long de la période de sa gestation? Cette question semble aller à l'encontre du sens commun, tant la grossesse est un événement marquant : le corps de la femme grossit et ses sensations sont encombrées par les signes physiques de la présence du nouvel être dans son ventre; l'accouchement s'impose toujours comme un fait bouleversant, en particulier avec la douleur parfois traumatisante qui l'accompagne. Dans le débat actuel, on parle fréquemment de « déni de grossesse ", catégorie incertaine de la nosographie psychiatrique qui désigne soit le symptôme psychique d'un état psychopathologique, soit un trouble de type psychosomatique ${ }^{2}$. Force est de constater, néanmoins, que la question de l'ignorance de la grossesse ne se pose pas que dans la clinique et le savoir psychologique d'aujourd'hui. Au 
début du XIXe siècle, en France, ce sont des médecins légistes qui, dans les premiers grands traités d'une discipline en pleine phase d'affirmation institutionnelle et professionnelle, abordent ce problème lorsqu'ils interrogent les savoirs médicaux sur la grossesse. La capacité des femmes à reconnaître l'état de grossesse surgit en effet à l'occasion des jugements des affaires d'infanticide, l'ignorance de l'état de grossesse est l'argument le plus souvent mobilisé par les prévenues pour se défendre contre l'accusation d'avoir volontairement mis à mort leur nouveau-nés. Dans le cadre des jugements pour infanticide, les discours, les narrations, les preuves concernant l'état et la période de la grossesse constituent, en effet, un moment très important ainsi qu'un matériau à soumettre à l'expertise des médecins. La médecine judiciaire, qui prend une importance croissante à la fin de l'âge moderne, devient un des principaux domaines qui font de l'observation de l'état de grossesse un objet de discussion et de production de savoirs. Cette discipline relie les savoirs médicaux et les lois qui régissent la société. Ainsi, avant la naissance d'une " psychologie de la puerpéralité », et dans un cadre intellectuel et pratique assez différent, les légistes s'interrogent sur les états psychiques de la femme enceinte, sur ce qu'elle sait ou ne sait pas de son propre corps.

2 La question de l'ignorance de la grossesse est donc au carrefour de la théorie médicolégale et du traitement judiciaire des affaires d'infanticide. Afin de mieux cerner les positions des médecins, nous avons mené une enquête sur les jugements pour infanticide rendus par le Tribunal de la Seine dans la deuxième moitié du XIX ${ }^{\mathrm{e}}$ siècle, en dépouillant les 80 dossiers de procédure pour les années 1865-1893 conservés dans les archives de la cour d'assises. Plusieurs acteurs interviennent sur cette scène judiciaire et confrontent leurs savoirs et leurs croyances: femmes prévenues, juges et médecins légistes. Si les savoirs médicaux influencent et déterminent les décisions finales des juges, la médecine légale - avec les enjeux qui lui sont propres dans cette période historique précise contribue aussi à l'émergence de nouveaux « objets scientifiques », tels que la psychologie de la femme enceinte, la grossesse et la subjectivité féminine.

\section{De l'incertitude}

3 Dans le cadre juridique qui définit l'infanticide à partir de l'âge moderne, et qui a ses origines dans le dispositif des déclarations de grossesse, le « recèlement de grossesse ${ }^{5}$ induit d'emblée un certain indice de culpabilité de la femme, car de ce "recèlement » l'accusation peut déduire son intention préméditée d'annuler l'existence du nouvel être dès ses premières manifestations. Ce même raisonnement justifie en partie la sévérité des peines établies par le Code pénal de 1810 : la loi napoléonienne punit l'infanticide - défini comme le « meurtre de l'enfant nouveau-né » (art. 300) - de la peine capitale (art. 302), en l'assimilant à l'assassinat, c'est-à-dire à un meurtre commis avec préméditation. Comme l'avocat et jurisconsulte Louis-Joseph Faure l'explique face au corps législatif, «il est impossible que l'infanticide ne soit pas prémédité ; il est impossible qu'il soit l'effet subit de la colère ou de la haine, puisqu'un enfant, loin d'inspirer de tels sentiments, ne peut inspirer que celui de la pitié ${ }^{6}$. Inversement, le fait d'avoir ignoré la grossesse représente une des excuses possibles pouvant jouer en faveur de la mère soupçonnée, la mort du nouveau-né ne pouvant plus alors découler d'un acte prémédité.

4 C'est pourquoi la loi sur l'infanticide évolue au cours du XIX ${ }^{e}$ siècle : la possibilité des circonstances atténuantes pour la mère qui se rend coupable de ce crime est introduite par la loi 25 juin 1824. Il s'agit d'un premier assouplissement du rigorisme légal 
napoléonien, qui introduit la possibilité d'une réduction de la peine par les magistrats des cours d'assises pour certains crimes parmi les plus graves. Cette disposition est étendue en 1832 à tous les crimes, en transférant la décision des magistrats vers le jury. Pour contrebalancer la rigueur de la loi, la justice s'assouplit, en admettant toutes ces circonstances qui peuvent susciter des doutes sur la culpabilité des prévenues, afin de leur épargner la peine capitale : «L'officier de police, dit Joseph Ortolan, doit recueillir avec plus grand soin toutes les circonstances qui peuvent établir, soit la culpabilité, soit l'innocence de la mère. Les charges les plus accablantes ne doivent jamais lui paraître suffisantes; tant qu'il lui reste encore quelques points, quelques circonstances à examiner, il ne doit rien rejeter comme inutile $»^{7}$. Le discours juridique devient beaucoup plus nuancé, il admet que « la dissimulation de la grossesse, les traces d'un accouchement clandestin, la découverte même du cadavre de l'enfant ne sont encore que des vagues indices du crime ; car, si la grossesse, si la naissance même ont été celées, la crainte de dévoiler sa honte suffit pour expliquer ce mystère dans une mère coupable $»^{8}$. Quelques décennies plus tard, en 1863, une loi modifie la définition du crime de suppression d'enfant (art. 345) de façon à permettre aux accusés d'infanticide d'obtenir une réduction de peine lorsqu'il n'est pas établi par l'expertise que l'enfant a vécu'.

5 Cette attitude de bienveillance est confirmée par les décisions de justice prises par la cour d'assises de la Seine pendant la période allant de 1867 à 1891 . Parmi les arrêts des 80 procès que nous avons examinés, il y a un nombre assez élevé d'acquittements : 81 sur le total des 84 accusés; la plupart sont des femmes : 36 prévenus; - 34 femmes et deux hommes, soit plus d'un tiers du nombre total -, sont acquittés par le jury, alors que les autres accusés sont condamnés de quatre mois à douze ans de prison ou de travaux forcés. Aucun accusé n'est condamné à la peine de mort ${ }^{10}$.

6 La plupart des femmes accusées d'infanticides se défendent en affirmant ne pas avoir eu conscience de leur grossesse. Des interrogatoires et des dépositions des témoins, il apparait que les femmes, au cours de leur grossesse, notamment quand il s'agit d'une grossesse illégitime, évitent les questions de leurs proches, dissimulent leur état physique, en s'habillant par exemple avec des vêtements pouvant masquer les rondeurs de leur corps. "Elle portait toujours un caraco et beaucoup de jupes $"^{11}$ dit Berthe Bonneau, la patronne de Marguerite Verdier, jugée en 1873 par le tribunal parisien. La maitresse de l'accusée déclare de surcroît avoir demandé à sa domestique la raison de la disparition de ses règles. La jeune femme s'abstient de répondre et proteste, en niant ce dont on l'accuse.

7 Soupçonnées par leur patron, leurs parents ou leurs voisins, les femmes se taisent ou cherchent des justifications concernant leur état physique anormal, qui suscite inévitablement la curiosité : les douleurs, l'absence des règles et l'augmentation du volume du corps. On déclare à propos d'Eugénie Theyssier que « dès les premiers jours mad. Borde remarque que sa servante avait la taille épaisse, elle lui en fait l'observation, mais celle-ci lui répondit que dans la maison où elle était employée précédemment elle était obligée de faire des travaux au dessus de ses forces et que le gonflement dont lui parlait sa maitresse n'avait pas d'autre origine $»^{12}$. Hortensia Jeunet réagit de la même manière : «Les voisines l'interpellèrent plusieurs fois à ce sujet mais elle nia toujours sa grossesse, disant aux unes qu'elle était hydropique, et aux autres qu'elle avait une tumeur dans l'abdomen $»^{13}$. Des états pathologiques sont souvent évoqués pour rendre compte des transformations physiques les plus évidentes, comme dans le cas de la disparition du cycle menstruel. Maria Gauge avoue ainsi avoir menti : «Comme je ne voulais pas qu'on 
sache ma grossesse et qu'elle commença à paraître, j'ai dit à madame que je n'avais pas mes règles, que j'avais déjà été comme cela à Château Thierry et que ça avait fini par des hémorragies, que ça serait peut être encore comme ça. C'est vrai qu'à Château Thierry j'avais eu un retard de cinq mois, mais alors je n'étais pas enceinte $»^{14}$.

Cette attitude de dénégation peut être tenue aussi face aux médecins. Ces derniers peuvent en effet, si l'on en croit les dépositions, ne pas contredire ouvertement les déclarations faites par leurs patientes. À cet égard, l'histoire de Marie Languinier est exemplaire : elle s'était rendue chez le médecin car « elle toussait beaucoup et ne voyait plus ses règles "; en lui relatant ses symptômes elle évita avec soin toute référence à une possible grossesse. Le médecin lui-même déclare à la justice qu'il lui avait demandé «si elle n'avait aucun motif pour expliquer l'absence de ses menstrues. Elle me répondit que non et accepta le traitement emménagogue (ferruginine, quinquina) que je lui prescrivis $»^{15}$.

9 Il est vrai que, dans la plupart de ces affaires, les prévenues finissent par avouer avoir trompé leur entourage et avoir caché leur grossesse. Elles expliquent leur comportement en quelques phrases brèves - synthèse lapidaire d'un interrogatoire probablement plus circonstancié - mais néanmoins assez significatives : «Je ne jugeais pas convenable de faire connaitre ma position à des étrangers ; je comptais alors aller faire mes couches soit à la maternité soit ailleurs $»^{16}$. «Je n'osais pas le dire car j'avais honte $»^{17}$, explique Marie Languinier. "Je ne pensais à rien, proteste Marie Gauge. Je ne pensais qu'à une chose, c'est que si mes patrons qui sont du pays savaient ça, ils le diraient à mes parents ${ }^{18}$.

Ceci étant dit, quelques accusées se présentent devant le tribunal en affirmant avec force avoir méconnu leur grossesse jusqu'à la fin. «Je n'ai pas pensé que je fusse enceinte » ${ }^{19}$. C'est par ces mots que Julia Marchand, une jeune fille accusée au cours de l'année 1875, se défend face au juge d'instruction. "Je ne me suis pas doutée sur le moment que j'accouchais » : elle affirme avoir été surprise par les douleurs de l'accouchement et pour cela ne pas avoir pu empêcher la mort immédiate de son enfant, advenue suite à la chute de celui-ci dans les latrines. L'année suivante, le même scénario se répète lors du procès contre Eugénie Voluchenendre : "Je ne me croyais pas enceinte », dit-elle. Elle résiste aux pressions exercées par le juge d'instruction: "Je vous assure que non. À la fin de septembre j'ai eu mes règles une fois, puis pendant quatre mois j'étais dans le sang ». Elle révèle, de plus, qu'elle était allée plusieurs fois chez le médecin car elle souffrait-d'une inflammation à l'estomac, mais « comme il lui demandait à mots couverts si elle n'avait point eu de relations avec un homme, elle se montra offensée ». L'accusée affirme ne pas avoir été consciente de sa grossesse jusqu'à la dernière minute de celle-ci : «Et vous n'avez jamais senti votre enfant remuer? » demande le juge ; « Non, jamais » ${ }^{20}$.

11 Bien évidemment, l'étude de ces déclarations impose de prendre en compte les contraintes imposées aux prévenues par le dispositif de l'interrogatoire judiciaire. Les dépositions des inculpées s'inscrivent dans des stratégies de défense, et l'incertitude concernant l'état de grossesse en est souvent la pierre angulaire. Si ces déclarations nous en disent très peu sur le vécu subjectif de la grossesse des accusées, elles nous renseignent, malgré tout, sur les représentations et les savoirs relatifs à l'état de grossesse, tant dans la vie quotidienne et familiale que dans la pratique médicale. La grossesse est un mystère, un phénomène caché par le corps féminin, soumis à la fois à des formes de contrôle - par exemple de la part des maîtresses des jeunes domestiques, soucieuses que la honte d'une grossesse illégitime ne se produise pas dans leur maisonet à la discrétion, à la circonspection dictées par les normes de la pudeur ${ }^{21}$. Ce 
phénomène, en partie secret et mystérieux, se donne à voir par nombreux signes : les douleurs, le grossissement du ventre, la suppression des règles, les mouvements du foetus. Ceux-ci, néanmoins, doivent être interprétés, voire repérés, afin de dévoiler ce qui se passe dans l'espace invisible à l'intérieur du ventre de la femme.

Cet aspect mystérieux se retrouve dans les principes du savoir obstétrique à partir du XVIII ${ }^{\mathrm{e}}$ siècle, dans les traités sur l'art des accouchements, utilisés pour l'instruction des sages-femmes ou de la communauté savante des médecins. Le savoir diagnostique de la grossesse est traversé par le thème, quasi obsessionnel, de l'incertitude. Celle-ci est renforcée par les difficultés rencontrées par les médecins qui doivent respecter la pudeur féminine durant l'examen ${ }^{22}$. Elle est liée aussi à la variabilité subjective des signes de grossesse : la difficulté à les repérer et à les interpréter correctement peut entraver le jugement et conduire à des erreurs. Les cas de fausses grossesses compliquent encore davantage la tâche, il s'agit de phénomènes souvent de nature pathologique dont les symptômes peuvent être confondus avec les signes de l'état gravidique. La fausse grossesse est constituée "par une môle, tantôt comme charnue, tantôt comme vésiculaire », ou encore par "ces amas de sang, d'eau, et d'humeurs glaireuses qui se forment dans la matrice, ainsi que la tympanite de ce viscère $»^{23}$. De tels cas sont documentés en grand nombre dans la littérature obstétrique des siècles précédents ${ }^{24}$.

Le savoir des accoucheurs vise à annuler, ou du moins, à réduire l'incertitude qui entoure la grossesse par la recherche de signes objectifs. Cet effort caractérise les développements des connaissances obstétriques tout au cours du XIX siècle ; l'invention du stéthoscope (en 1819 par Laennec) et l'introduction de la technique de l'auscultation fœtale (mise au point par Jean-Alexandre de Kergaradec en 1822) permettent désormais d'avoir un signe spécial, ensuite considéré comme le seul véritablement certain, de la grossesse: le battement du cœur du foetus ${ }^{25}$. Seul ce signe peut révéler l'existence d'un autre être vivant au sein de la femme et seul le médecin, grâce à sa technique et à ses compétences, peut l'obtenir. En revanche, la parole et le vécu des femmes sur leur corps - comme la perception des mouvements du fotus - ne sont considérés que comme des symptômes subjectifs peu utiles pour le médecin.

14 Ainsi, malgré la prolifération des études et des traités, malgré l'invention de nouveaux instruments, le diagnostic de grossesse reste un problème irrésolu et la grossesse une condition incertaine. Les questions relatives aux "grossesses douteuses", aux "grossesses méconnues" et aux "erreurs ${ }^{26}$ de diagnostic restent en effet un thème largement débattu par les obstétriciens du XIXe siècle. Des signes auparavant considérés comme irréfutables semblent maintenant plus douteux, si on les compare à la certitude produite par la technique de l'auscultation. Même « les mouvements perçus par la femme comme produits par le fotus doivent toujours être accepté par le médecin sous bénéfice d'inventaire. L'accoucheur seul a qualité pour apprécier la véritable nature des mouvements ressentis $»^{27}$. En effet la parole et les sensations de la femme perdent de sa valeur dans le procédé de connaissance du phénomène. Elle peut facilement se tromper, et surtout elle peut mentir ou cacher des détails.

\section{Le juge ou le médecin?}

15 "Quelques auteurs ont prétendu le fait qu'à raison de l'incertitude des signes de la grossesse, une femme enceinte pouvait ignorer son état, surtout si cette grossesse n'avait pas été précédée par d'autres, qui puissent lui donner quelque expérience. $»^{28}$ Cette 
réflexion du médecin légiste Paul-Augustin-Olivier Mahon au début du XIX ${ }^{\mathrm{e}}$ siècle, soulève une question qui restait auparavant étrangère à la pratique médico-judiciaire française. Si à l'époque moderne, la grossesse constituait l'un des enjeux les plus fréquents des examens des experts des tribunaux, l'observation se bornait à l'inspection anatomique de la femme à juger, afin de reconnaître l'existence d'un tel état et, éventuellement, l'époque de son développement ${ }^{29}$. Mais la médecine légale connaît des transformations importantes après la Révolution. Elle devient une discipline autonome avec la loi de 1803 et se dote de chaires d'enseignement dans les principales universités européennes $^{30}$. Mahon est le premier titulaire de celle de Paris et sans doute le premier, au moins en France, à prendre comme objet d'investigation non pas les états physiques de la femme enceinte mais, au contraire, ses états psychiques, c'est-à-dire la connaissance qu'elle pouvait avoir de sa propre grossesse.

Comment aborder la question? Il faut reconnaître, selon Mahon, qu'une femme peut ignorer avoir conçu. Les médecins savent désormais que, contrairement à ce qu'on croyait autrefois, la conception n'est pas la conséquence du plaisir éprouvé par la femme pendant le rapport sexuel ; il est donc possible qu'une femme reste enceinte sans avoir eu aucune notion, perception ou souvenir de l'acte sexuel qui en est à l'origine. On trouve d'ailleurs de tels exemples dans la littérature : dans les Essais de Michel de Montaigne, narrant l'histoire d'une paysanne qui, pendant un profond sommeil provoqué par l'ivresse, est engrossée par son valet; ou encore dans La Marquise d'O. de Heinrich von Kleist, nouvelle publiée en 1808 , l'héroïne ayant été violée par un officier russe alors qu'elle avait perdu conscience ${ }^{31}$. Si donc une femme ignore avoir eu un rapport sexuel - parce qu'elle est inexperte, ou parce qu'elle dormait, ou, magnétisée, était en état de somnambulisme magnétique (hypnose), ou était prise par les convulsions ou dans un état d'ivresse - elle peut tout aussi bien, selon Mahon, en ignorer les conséquences ${ }^{32}$.

En posant la même question, quelques années plus tard, le légiste strasbourgeois François-Emmanuel Fodéré se demande : «l'accusée a-t-elle pu ignorer sa grossesse et la confondre avec une autre maladie $»^{33}$ ? Il évoque d'abord «l'incertitude des signes d'une grossesse commençante et la facilité qu'il y a à la confondre avec toute autre maladie » et, par la suite, «l'autorité » des illustres médecins et accoucheurs qui ont documenté la possibilité de telles erreurs. Néanmoins Fodéré, tout comme Mahon, ne cache pas son scepticisme vis-à-vis de ce type de cas très exceptionnels. Car cette éventualité ne se pose, selon lui, que si la femme est complètement idiote, ou si, pour d'autres raisons - ivresse, affections comateuses ou asphyxie - ses facultés mentales sont annulées, au point qu'elle n'a pu soupçonner avoir été fécondée.

18 La question telle qu'elle est soulevée par Mahon ou Fodéré reste toutefois sur un plan purement théorique et général : la discussion occupe peu de pages dans leurs traités, ils présentent les cas les plus célèbres, mais sans donner d'instructions pratiques sur le type d'observation, ou d'examen, à effectuer dans les cas où une femme accusée d'infanticide opposerait une telle revendication. Une question se pose davantage: qui doit se prononcer sur cette prétendue ignorance, donc sur les états psychiques, de la femme inculpée ? Cela relève-t-il de la compétence du médecin, étant donné que cette question surgit à partir de faits et d'observations médicales, ou de la compétence de la justice, véritable juge des intentions des hommes (et des femmes) criminels? Chez les auteurs cités, cela n'est ni clair, ni explicite.

19 En 1840, Alphonse Devergie, expert auprès des Tribunaux de la Seine, approfondit cette question dans son ouvrage Médecine légale, théorique et pratique. Après avoir exposé les 
moments de l'examen du corps de l'accusée, Devergie précise que la tâche de formuler un jugement sur l'ignorance de la grossesse revient à la justice et non pas au médecin : « ces donnés étant plutôt du ressort de l'appréciation des jurés que des médecins experts, il nous suffit d'avoir fait connaître les raisons qui militent pour et contre et d'avoir établi la possibilité qu'une femme ignore sa grossesse $»^{34}$. Mais le problème reste ouvert pendant les décennies suivantes.

\section{L'ignorance : un « objet » scientifique}

« Où est la naïve enfant qui, de nos jours, peut prétexter de son ignorance jusqu'à la fin? À moins d'idiotie complète, cela n'est plus admissible, pas plus que l'accouchement sans le savoir ». Ainsi Chatagnier, juge d'instruction à Bourg, auteur d'un ouvrage très sévère sur le crime des mères meurtrières, s'adresse à ses lecteurs et collègues, en les mettant en garde contre les prétendues naïvetés des prévenues qui allèguent ne pas avoir été conscientes de leur grossesse. Pour lui, les signes de cet état sont très clairs: « La suppression des menstrues, le développement de l'abdomen et des seins, commencent à l'effrayer ; puis, vers le milieu de la gestation, les mouvements du foetus, son ballotement, et l'ampleur toujours plus développée de son ventre et de ses mamelles, ne lui permettent normalement plus de doute sur son état de grossesse $\aleph^{35}$.

21 Cependant, les positions des légistes de la même époque n'affichent pas la même fermeté quand il s'agit d'aborder ce problème. En effet, la question de l'ignorance de la grossesse chez la femme enceinte revient ponctuellement dans les ouvrages médico-légaux de la deuxième moitié du XIX ${ }^{e}$ siècle, où l'on commence à traiter le sujet sous un angle nouveau. En 1867, un ouvrage d'Auguste Ambroise Tardieu, titulaire de la chaire de médecine légale de la faculté de Paris, porte ainsi exclusivement sur l'infanticide. Tardieu adopte une approche sensiblement différente de ses prédécesseurs: tout en reconnaissant la possibilité que cela puisse arriver, « il ne s'agit pas - précise Tardieu - de dire si une femme, peut, en thèse générale, ignorer sa grossesse ${ }^{36}$. Au contraire, selon Tardieu, le médecin expert doit savoir faire face à une telle difficulté de façon concrète, en fonction des cas singuliers. L'expert, dit l'auteur, « n'a à juger que la question de fait et à l'apprécier dans ce qu'elle a d'actuel $\aleph^{37}$. En tenant compte des caractéristiques physiques et physiologiques du sujet, de la conduite de la femme au cours de la grossesse, qui ne relèvent pas nécessairement de la compétence du savoir médical, le médecin peut formuler un jugement qui, s'il n'est pas tout à fait certain, peut être du moins vraisemblable ${ }^{38}$.

Près de 20 ans après l'ouvrage de Tardieu, Gabriel Tourdes, titulaire de la chaire de médecine légale à Nancy et doyen de la Faculté de médecine, propose une des plus riches réflexions sur la question dans son article «Grossesse-Médecine Légale » du Dictionnaire encyclopédique des sciences médicales publié en 1886. En écho au propos de Tardieu, il précise que « dans chaque cas particulier l'expert recherche les conditions qui lui permettent d'établir que cette ignorance était réelle ou simulée " ${ }^{39}$. C'est donc l'expert qui décide si l'ignorance de la prévenue est réelle ou simulée. "Nous examinerons d'abord l'état mental de la femme, écrit le légiste; l'idiotie, un trouble permanent de l'intelligence, ont pu empêcher, altérer les perceptions $»^{40}$. Après l'appréciation de l'état mental, Tourdes préconise un examen physique et physiologique de la femme, avant de juger la conduite de la prévenue: «A-t-elle dissimulé son état? A-t-elle réclamé des secours médicaux, en parlant de la suppression des règles? A-t-elle fait usage des moyens 
abortifs ? $»^{41}$. Le but de l'examen médico-légal, dont Tourdes donne les instructions, est clair : il ne s'agit pas simplement de fournir des observations sur l'état de l'accusée, pour constater si des phénomènes physiologiques ou pathologiques ont pu être pris pour une grossesse, ni d'exposer des raisons ou des connaissances théoriques qui peuvent tout simplement éclairer la décision des jurés. Il s'agit au contraire d'établir si la prévenue ment ou dit la vérité et de produire un jugement ponctuel sur ce qu'elle sait ou ne sait pas, ceci en donnant beaucoup d'importance à « la moralité de la femme, l'intérêt qu'elle avait à cacher sa grossesse, sa conduite au moment de l'accouchement", autant d'éléments qui n'appartiennent pas nécessairement au domaine des connaissances médicales. On peut souligner comment le discours médico-légal ici déployé dénote les contours d'un objet d'enquête qui est propre au dispositif judiciaire, à savoir "l'intériorité » du criminel ${ }^{42}$, reconstruite à travers le rassemblement minutieux de la totalité des circonstances qui entourent l'action criminelle. Cette intériorité est désormais appréhendée, par Tardieu et par Tourdes, comme un objet de prédilection du savoir médico-légal, un objet spécifique d'un savoir qui se constitue dorénavant en tant que « science » du crime et du criminel ${ }^{43}$.

Cette opinion n'est pas sans rapport, bien sûr, avec les contributions des aliénistes sur la question de l'irresponsabilité pénale, même si cette question ne porte pas, strictement parlant, sur la responsabilité du prévenu. Elle porte plutôt sur les éléments psychiques qui composent les intentions du sujet, sur ce qu'il, ou elle, peut ou ne peut pas connaître ou reconnaître : ainsi la psyché est objectivée par le biais des observations sur le corps, ses caractéristiques, ses fonctions et ses conduites. Si la femme naïve et ignorante semble ne plus exister selon le juge, l'ignorance de celle-ci existe en tant qu'objet d'enquête scientifique. Ce qui n'implique pas nécessairement une expertise psychiatrique mais un examen qui, comme nous avons essayé de le montrer, n'est pas vraiment définissable ni en termes médicaux ni en termes juridiques.

\section{Le tribunal : un lieu de circulation des savoirs sur la grossesse}

Avant de clore cette analyse de la littérature médico-légale, il convient de s'intéresser au Précis de médecine légale du médecin légiste Charles Vibert, écrit en 1886. Vibert était alors expert depuis six ans auprès du tribunal de la Seine : c'est à lui que furent confiées la plupart des expertises, notamment celles relatifs aux affaires d'infanticide examinées ici. Comme Alphonse Devergie, mais à la différence de la plupart des autres auteurs précédemment cités, Vibert n'est pas titulaire d'un enseignement universitaire : il est maître des conférences pratiques de médecine légale à la morgue de Paris, où il enseigne aux étudiants la pratique des autopsies judiciaires et les théories issues des travaux et des recherches du laboratoire de la morgue ${ }^{44}$. L'ouvrage qu'il rédige en 1886 est un véritable manuel professionnel, «écrit par un expert pour des experts » ${ }^{45}$, comme le dit son illustre collègue Paul Brouardel, à l'époque titulaire de la chaire de médecine légale de la Faculté de médecine de Paris, dans la préface de l'ouvrage.

Dans ce Précis, qui connaît un grand succès (il sera réédité dix fois, sa dernière publication, posthume, datant de 1921, après la mort de son auteur), la question de l'ignorance de la grossesse chez la femme enceinte est traitée de manière très concise, dans l'espace d'une seule page. «Ces cas sont en petit nombre, souligne Vibert, et, d'une 
façon générale, il n'y a guère lieu de tenir compte de ces exceptions si peu souvent constatées ». De plus, selon lui, le peu d'importance que le médecin légiste doit accorder à ces exceptions tient au fait que, comme il l'écrit, la question elle-même ressort de la compétence de la justice et non pas du médecin expert : « encore est-ce aux magistrats ou aux jurés qu'il appartient de décider si une fille a pu être assez simple et assez ignorante pour ne pas connaître les conséquences possibles du coït auxquelles elle s'est livrée, et pour ne pas comprendre la signification des phénomènes qui se passaient en elle $»^{46}$.

Le propos de Vibert est assez surprenant, car il semble aller à contre-courant des positions défendues par Tardieu et Tourdes lorsqu'ils élargissent le champ de compétence du médecin légiste en lui confiant la tâche du jugement sur les éléments psychiques de la prévenue. Vibert, au contraire, semble ici faire un pas en arrière, en redonnant aux juges - magistrats et jurés - la faculté de se prononcer sur cet objet disputé entre justice et médecine. Force est de constater, en effet, que l'émergence d'un domaine spécifique, relevant de la compétence du savoir médico-légal, à savoir l'«intériorité » de l'accusée, l'ensemble des états psychiques et de ses intentions, permettant de déduire l'ignorance ou la conscience de la grossesse chez celle-ci, est lié à l'effort des auteurs des écrits médico-légaux pour faire de leur discipline une "science", un savoir distinct et autonome qui, comme tel, doit se doter d'« objets » d'observation et de jugement qui lui sont propres. Les textes de Tardieu et de Tourdes répondent à cette vocation, car il s'agit d'ouvrages (ou de parties d'ouvrages) théoriques, universitaires, qui s'adressent à la communauté savante des médecins et pas seulement aux médecins experts. Ces deux écrits se situent donc à l'intérieur du processus de constitution de la discipline médicolégale en tant que «science médicale ». Inversement, l'ouvrage de Vibert est au contraire un manuel professionnel, « un traité concis, dans lequel il trouve le bilan actuel de la science, l'interprétation des signes qu'il rencontre et qui se rapportent aux cas les plus ordinaires de la criminalité $\aleph^{47}$. Dans ce texte, il est donc davantage question des enjeux propres à la profession des experts des tribunaux et à leur rôle face à la justice, que des contributions des universitaires et des savants à l'ensemble des connaissances médicales. Et dans ce contexte, la question de l'ignorance de la grossesse chez la femme trouve une place très réduite.

Le propos de Vibert éclaire les documents d'archives des jugements de la cour d'assises que nous avons consultés. On peut en effet constater que, parmi les 80 procès conservés dans les archives du Tribunal de la Seine, l'expert n'est jamais interrogé sur la possibilité qu'une des prévenues ait pu ignorer sa propre grossesse. Ce sont donc, comme Vibert le reconnait, les juges, et surtout les juges d'instruction, qui gardent la compétence de ce type d'appréciation autour des états psychiques et des intentions des accusées, et notamment de l'examen de leurs conduites qui sont interrogées, discutées et mises à l'épreuve lors des interrogatoires. La même circonspection et attention que les médecins préconisent à leurs élèves (ou collègues) caractérise la posture des magistrats face aux déclarations des prévenues. De surcroit, les juges d'instruction semblent bien connaître les «mystères" de la grossesse, car, pendant les interrogatoires, ils n'hésitent pas à mettre les accusées sur la sellette, en donnant preuve d'une certaine compétence (et d'un savoir certain), sur ce qui concerne les signes de cet état exclusivement féminin : les règles, les mouvements de l'enfant, l'époque où les signes deviennent plus évidents, les douleurs de l'accouchement, parfois aussi leur intensité. «Les préliminaires d'un accouchement - atteste le juge en questionnant la mère de l'inculpée Julia Marchand d'un premier accouchement surtout, ne sont point aussi simples que vous voudriez le 
faire croire. Il y a les petites douleurs, les grandes douleurs et vous avez assisté à toute cette scène $\aleph^{48}$. Sans doute grâce à l'affirmation et à la circulation du savoir médical de la grossesse, notamment dans les tribunaux, et dans les ouvrages de médecine légale dont les magistrats aussi sont les destinataires, les juges montrent qu'ils savent mobiliser les connaissances des médecins au profit de la justice. «Peut-être dans les premiers temps, mais après 6 ou 7 mois, vos doutes ont dû cesser »: assène le juge à l'attention de l'accusée Julia Marchand au sujet de sa "grossesse ignorée ». Celle-ci finit par avouer: «c'est vrai, j'ai fini par penser que je devais être enceinte " $^{49}$.

Ainsi, les juges font preuve d'une certaine méfiance vis-à-vis des déclarations des accusées, dont la parole est défiée et souvent démentie au cours des interrogatoires. Ceci semble néanmoins ne pas influencer les décisions finales prises par la justice, car Julia Marchand, tout comme beaucoup d'autres accusées, est acquittée par la cour d'assisses. Les juges et les jurés se montrent cléments dans leurs sentences concernant ce type de crime, comme s'ils se révélaient sensibles à l'inexpérience, la naïveté ou l'ignorance des accusées.

La question de l'ignorance de la grossesse chez la femme enceinte reste, pendant tout le $\mathrm{XIX}^{\mathrm{e}}$ siècle, une question strictement médico-légale, c'est-à-dire un problème à la fois médical, en ce qu'il implique des connaissances sur le corps et la physiologie de la femme enceinte, et juridique, car il impose d'interroger les intentions criminelles des femmes infanticides. Ceci étant dit, l'évolution des réponses données par les médecins légistes est donc liée aux progrès de cette discipline, à son institutionnalisation en tant que matière d'enseignement, à sa reconnaissance en tant que science. L'ignorance de la grossesse est en effet un « objet » scientifique propre à cette discipline en train de se constituer au sein des sciences médicales, néanmoins elle trouve très peu de place dans la pratique des experts des tribunaux. Bien sûr, les développements de l'obstétriques au cours du XIX ${ }^{\mathrm{e}}$ siècle, notamment en ce qui concerne le savoir diagnostique de la grossesse, interrogent les magistrats: le médecin peut produire une connaissance de la grossesse plus certaine que celle qui est ressentie par la femme elle-même, et donc, à plus forte raison, que celle qui est soupçonnée ou présumée par les juges des tribunaux. De plus, face au médecin la femme est un sujet « ignorant » : elle n'a pas accès à la certitude de son état de grossesse, car les signes qui l'attestent peuvent être attestés seulement par le médecin et par les procédés techniques dont il dispose, tels que, par exemple, la technique de l'auscultation fœtale.

30 Mais les progrès des savoirs en obstétrique ne suffisent pas, la psychiatrie doit aussi être prise en compte. Fodéré et, ensuite, Tourdes, soulignent l'importance de certains états mentaux pathologiques, tels que par exemple l'idiotie, qui peuvent troubler les perceptions et les facultés du sujet. Néanmoins, il n'y a pas de référence dans les ouvrages des experts devant les tribunaux aux théories de l'époque sur les folies puerpérales, y compris celle des folies dites des femmes enceintes ${ }^{50}$, pas plus qu'au sujet-des femmes maniaques qui accouchent sans s'en apercevoir, ayant oublié avoir été enceintes à cause de l'abolition de la sensibilité à la douleur provoquée par la manie ${ }^{51}$. Quant aux femmes ivres, magnétisées et en état hypnotique, qui deviennent enceintes à leur insu, si les médecins légistes du début du siècle citent ponctuellement ces exemples - sans doute nourris par l'imaginaire littéraire de l'époque - les références disparaissent dans la 
deuxième moitié du siècle. Si l'histoire du psychisme au XIX ${ }^{e}$ siècle est riche de ces récits de maternité inconsciente de ses origines sexuelles et physiques ${ }^{52}$, les médecins légistes semblent en tenir de moins en moins compte. On ne saurait pas ici donner plus d'explications de cette disparition, qu'on peut sans doute lier en partie au discrédit jeté par la médecine académique aux phénomènes magnétiques autour de la moitié du siècle. Dans ce sens il faudrait creuser dans les rapports que la médecine légale entretient avec le renouveau du débat sur les états hypnotiques prenant une grande ampleur dans les années 1870, ainsi qu'avec les nouvelles perspectives ouvertes par le paradigme neurologique de l'hystérie autour de la sensibilité et de la sexualité féminines ${ }^{53}$.

En tout cas, si l'on considère cette figure médico-littéraire de la femme victime passive et inconsciente de l'action d'autrui sur son propre corps, l'image de la femme que l'on retrouve chez les médecins légistes qui s'interrogent sur l'ignorance de la grossesse est le plus souvent presque antithétique: il s'agit d'une femme rusée, qui dissimule son état physique pour tromper son entourage et se livrer au crime. Ce sujet paradoxal, à la fois ignorant - en tant que femme et en tant qu'étrangère aux techniques et savoirs médicaux ${ }^{54}$ - et rusé - en tant que délinquante - constitue l'un des « objets » les plus controversés pour les sciences de l'homme au XIX ${ }^{e}$ siècle.

\section{NOTES}

2. Sophie Marinopoulos et Israël Nisand, Elles accouchent et ne sont pas enceintes : le déni de grossesse, Mayenne, Les liens qui libèrent, 2011 ; Marion Seibert, Le déni de grossesse. Enjeux et acteurs de la publicisation d'une cause secondaire, Paris, L'Harmattan, 2014.

3. Mark Jackson, "Something More than Blood": Conflicting Accounts of Pregnancy Loss in Eighteenth-Century England', in R. Cecil (ed.), The Anthropology of Pregnancy Loss, Oxford and Washington, Berg Publishers, 1996, p. 197-214 ; Laura Gowing, 'Secret births and infanticide in 17 th-century England', Past \& Present, vol.156, 1997, p. 87-115 ; Annick Tillier, Des criminelles au village. Femmes infanticides en Bretagne, 1825-1865, Rennes, Presses universitaires de Rennes, 2001 ; Silvia Chiletti, «Gravidanze nascoste. Narrazioni del corpo femminile nei processi per infanticidio tra Otto e Novecento », Genesis. Rivista della società italiana delle storiche, tome XII, $\mathrm{n}^{\circ} 1,2013$, p. 139-160.

4. On peut sans doute rapprocher l'émergence d'une psychologie de l'état puerpéral aux études d'Esquirol, et ensuite de Louis Victor Marcé, sur les aliénations des femmes grosses et accouchées et des nourrices : Étienne Esquirol, «De l'aliénation mentale des nouvelles accouchées et des nourrices ", Annuaire Médico-Chirurgical des Hôpitaux de Paris, tome IV, n 1, 1818, p. 600-632 ; Louis Victor Marcé, Traité de la folie des femmes enceintes des nouvelles accouchées et des nourrices et considérations médico-légales qui se rattachent à ce sujet, Paris, Baillières, 1858.

5. Les déclarations de grossesse sont un dispositif établi par l'Édit d'Henri II de 1556 visant au contrôle du corps maternel. Selon cette loi, toute femme enceinte est obligée de déclarer sa grossesse aux autorités ; si une femme n'a pas déclaré sa grossesse et que son enfant est retrouvé mort et privé du baptême, elle est condamnée à mort. cf. Marie-Claude Phan, "Les déclarations de grossesse en France, $\mathrm{XVI}^{\mathrm{e}}-\mathrm{XVIII}{ }^{\mathrm{e}}$ siècles", Revue d'histoire moderne et contemporaine, tome XXII, 1975, p.61-88. Sur la notion de «recèlement de grossesse », cf. Antoine Bruneau, 
Observations et maximes sur les matières criminelles : avec des remarques tirées des auteurs, conformes aux édits, ordonnances, arrêts \& reglemens [sic] des Cours souveraines. Ouvrage nécessaire à tous Juges, Avocats, Procureurs, Greffiers, huissiers et praticiens pour bien faire et instruire un procès criminel, Paris, Cavalier, 1715.

6. Code des délits et des peines, servant de supplément au procès-verbal des séances du corps législatif, Paris, Hacquart, 1810.

7. Joseph-Louis-Elzéar Ortolan, Le Ministère public en France : traité et code de son organisation, de sa compétence et de ses fonctions dans l'ordre politique, judiciaire et administratif, Paris, Fanjat, 1831, tome 2, p. 70.

8. Adolphe Chauveau et Faustin Hélie, Théorie du Code pénal, Paris, Legrand et Descauriet, 1837-1842, tome 3, p. 399. Pour une lecture intéressante des usages du droit pénal au XIX ${ }^{\mathrm{e}}$ siècle je renvoie à Laurence GUIGNARD et Gilles MALANDAIN, "Introduction : usages du droit dans l'historiographie du XIX siècle », Revue d'histoire du XIXe siècle, tome 48, n 1, 2014, p. 9-25.

9. Cf. Annick Tillier, Des criminelles au village..., op. cit., p. 30-31.

10. La moyenne des peines correspond à environ cinq ans de prison ou de travaux forcés. Plusieurs études se sont intéressées au phénomène de l'indulgence de la part des jurés des cours d'assises dans les affaires d'infanticide, cf. Dominique Vallaud, «Le crime d'infanticide et l'indulgence des cours d'assises en France au XIX ${ }^{\mathrm{e}}$ siècle ", Information sur les sciences sociales, tome XXI, n³, 1982, p. 475-499; Richard Lalou, "L'infanticide devant les tribunaux français (1825-1910) », Communications, vol. 44, 1986, p. 175-200 ; James M. Donovan, 'Infanticide and the juries in France, 1825-1913', Journal of Family History, vol. 16, n 2, 1991, p. 157-176; Simone Geoffroy-Poisson, «L'infanticide devant la cour d'assises de la Haute-Marne au XIX ${ }^{\mathrm{e}}$ siècle », Les Cahiers du Centre de Recherches Historiques, $n^{\circ}$ 35, avril 2005, p. 129-148.

11. Arch. dép. Seine, D2U8 21.

12. Arch. dép. Seine, D2U8 192.

13. Arch. dép. Seine, D2U8 149.

14. Arch. dép. Seine, D2U8 120.

15. Arch. dép. Seine, D2U8 52. Sur l'utilisation des emménagogues, cf. Agnès Fine, « Savoirs sur le corps et procédés abortifs au XIX ${ }^{\mathrm{e}}$ siècle ", Communications, tome 44, 1986, p. 107-136; Etienne Van de Walle et Elisha P. Renne, Regulating menstruation: beliefs, practices, interpretations, Chicago, University of Chicago Press, 2001.

16. Arch. dép. Seine, D2U8 21.

17. Arch. dép. Seine, D2U8 52.

18. Arch. dép. Seine, D2U8 120.

19. Arch. dép. Seine, D2U8 35.

20. Arch. dép. Seine, D2U8 48.

21. Cf. Emmanuelle Berthiaud, "Le vécu de la grossesse en France aux XVIII ${ }^{\mathrm{e}}$ et XIX ${ }^{\mathrm{e}}$ siècles", Histoire, médecine et santé, $n^{\circ} 2,2012$, p. 93-108. Sur les narrations des vécus de la grossesse dans les procès pour infanticide, cf. Annick Tillier, Des criminelles au village..., op. cit. 2001 et, pour l'Italie, Silvia Chiletti, « Gravidanze nascoste ... », loc. cit.

22. Cf. Hélène Rouch, Préface à Philippe Hecquet, De l'indécence aux hommes d'accoucher les femmes suivi de De l'obligation aux mères de nourrir leurs enfants, Paris, Côté Femmes, 1990 [1708] ; Nahema Hanafi, "Pudeurs des souffrants et pudeurs médicales, $\mathrm{XVI}^{\mathrm{e}}-\mathrm{XX}^{\mathrm{e}}$ siècle ", Histoire, médecine et santé, $\mathrm{n}^{\circ}$ 1, 2012, p. 9-18.

23. Idem, p. 208.

24. Cf. Cathy McClive et Helen King, 'When is a foetus not a foetus ? Diagnosing false conceptions in Early Modern France', in Véronique Dasen [dir.], L'embryon humain à travers l'histoire. Image s, savoirs et rites, Paris, Infolio, 2004, p. 223-238; Maria Conforti, " "Affirmare quid intus sit divinare est" mole, mostri e vermi in un caso di falsa gravidanza di fine seicento ", Quaderni storici, tome XLIV, $\mathrm{n}^{\circ} 1,2009$, p. 125-151. 
25. Cf. J.-A. De Kergaradec, Mémoire sur l'auscultation appliqué à l'étude de la grossesse, Paris, 1822 ; Jean-Anne-Henri Depaul, Traité théorique et pratique d'auscultation obstétricale, Paris, Labé, 1847.

26. Cf. Wilhelm Joseph Schmitt, Recueil d'observations sur des cas de grossesses douteuses, précédé d'une introduction critique sur la manière d'explorer, traduit de l'allemand par J. A. Stoltz, Strasbourg, Silbermann, 1829; Antoine Barbaroux, Des Grossesses méconnues, étude clinique et diagnostique, thèse de la Faculté de médecine de Montpellier, 1901 ; Charles Pajot, Des Causes d'erreur dans le diagnostic de la grossesse, Paris, Doin, 1874.

27. Charles Pajot, Des causes d'erreur..., op. cit., p. 14. C'est moi qui souligne.

28. Paul-Augustin-Olivier Mahon, Médecine légale et police médicale, Paris, Buisson, 1801, p. 379.

29. Michel Porret, "Sage-femme, chirurgien, médecin: les légistes de l'Ancien Régime, auxiliaires de justice », in Claire Dolan [dir.], Entre justice et justiciables : les auxiliaires de la justice du Moyen Age au XXe siècle, Sainte Foy, Presses universitaires de Laval, 2005, p. 719-735 ; Silvia De Renzi, 'Medical Expertise, Bodies and the Law in Early Modern Courts', Isis, tome XCVIII, $\mathrm{n}^{\circ} 2,2007$, p. 315-22.

30. Fréderic Chauvaud, Les experts du crime : la médecine légale en France au XIXe siècle, Paris, Aubier, 2000.

31. Cf. Mary Jacobus, 'In Parenthesis: Immaculate Conceptions and Feminine Desire', in Mary Jacobus, Evelyn Fox Keller, Sally Shuttleworth (eds), Body/Politics : Women and the Discourses of Science, New York, Routledge, 1990, p.11-28; on peut aussi signaler l'histoire d'une femme tombée enceinte après avoir été magnétisée, présentée dans le roman de Frédéric Soulié, Le magnétiseur (1834), cité par Jacqueline Carroy, «Immaculées conceptions », Nouvelle revue de psychanalyse, tome XLVI, 1992, p. 175-190.

32. Paul-Augustin-Olivier Mahon, Médecine légale..., op. cit., p. 380.

33. François-Emmanuel Fodéré, Traité de médecine légale et d'hygiène publique, Paris, Mame, 1813, tome I, p. 490.

34. Alphonse Devergie, Médecine légale, théorique et pratique, Paris, Baillière, 1840, p. 53.

35. Chatagnier, De l'infanticide dans ses rapports avec la loi, la morale, la médecine légale et les mesures administratives, Paris, Cosse, 1855, p. 214-215.

36. Auguste-Ambroise Tardieu, Étude médico-légale sur l'infanticide, Paris, Baillière, 1867, p. 218. Sur une certaine rivalité entre Tardieu et Devergie, cf. Bruno Bertherat, « L'élection à la chaire de médecine légale à Paris en 1879 », Revue historique, nº 644, 2007, p. 823-885.

37. Idem, p. 221.

38. Ibidem.

39. Gabriel Tourdes, « Grossesse - Médecine Légale », in Amédée Dechambre [dir.], Dictionnaire encyclopédique des sciences médicales, Quatrième série, tome 11, Paris, Masson, 1886, p. 306. C'est moi qui souligne.

40. Idem, p. 308

41. Idem, p. 309.

42. Laurence Guignard, "Les lectures de l'intériorité devant la justice pénale au XIX ${ }^{\mathrm{e}}$ siècle ", Romantisme, tome CXLI, numéro 3, 2008, p. 23-35; Michel Foucault, Surveiller et punir : naissance de la prison, Paris, Gallimard, 1975.

43. Marc Renneville, Crime et folie : deux siècles d'enquêtes médicales et judiciaires, Paris, Fayard, 2003 ; Laurence Guignard, Juger la folie. La folie criminelle devant les Assises au XIXe siècle, Paris, Presses Universitaires de France, 2010.

44. Cf. Bruno Bertherat, « L'élection à la chaire de médecine légale à Paris en 1879 »..., loc. cit.

45. Paul Brouardel, Préface, dans Charles Vibert, Précis de médecine légale, Paris, Baillière, 1886, p. IX.

46. Charles Vibert, Précis de médecine légale, op. cit., p. 353.

47. Paul Brouardel, Préface, op. cit, p. VIII. 
48. Arch. dép. Seine, D2U8 35.

49. Ibidem.

50. Cf. Louis Victor Marcé, Traité de la folie..., op. cit. Sur les folies puerpérales cf. Francesca Arena, « La maternité : entre santé et pathologie. L'histoire des délires puerpéraux entre époque moderne et contemporaine ", Histoire, médecine et santé, n³, 2013, p. 101-113.

51. Cf. Louis Victor Marcé, Traité de la folie..., op. cit., p. 93-94 ; Pierre Berthier, « Observation sur un délire mélancolique déterminé par une grossesse, passée inaperçue de tous, même de la malade, et jugée par un accouchement et une délivrance simultanés, également accomplis à l'insu de la malade ", Annales de la société impériale de médecine de Lyon, tome VII, $\mathrm{II}^{\mathrm{e}}$ série, 1859, p. 66-69.

52. Cf. Mary Jacobus, 'In Parenthesis...', loc. cit. ; Jacqueline Carroy, «Immaculées conceptions », loc. cit.

53. Cf. Georges Gilles de la Tourette, L'hypnotisme et les états analogues au point de vue médicolégal, Paris, Plon, 1887, p. 488 et suiv. cité par Nicole Edelman, «Médecine, expertise et genre : l'“affaire Castellan" ( $\mathrm{xIX}{ }^{\mathrm{e}}$ siècle) ", Histoire, médecine, santé, $\mathrm{n}^{\circ} 3$, 2013. Voir aussi Nicole Edelman, Les métamorphoses de l'hystérique. Du début du xixe siècle à la Grande Guerre, Paris, La Découverte, 2003.

54. Cf. Jacqueline Carroy, Nicole Edelman, Annick Ohayon et Nathalie Richard, Les femmes dans les sciences de l'homme (XIXe-XXe siècles) : inspiratrices, collaboratrices ou créatrices ?, Paris, Seli Arslan, 2005.

\section{RÉSUMÉS}

$\mathrm{Au}$ tournant des $\mathrm{XVIII}^{\mathrm{e}}$ et $\mathrm{XIX}{ }^{\mathrm{e}}$ siècles, en France, la grossesse est un des objets de réflexion privilégiés de la médecine légale. À partir de cette époque, cette nouvelle discipline - où l'on voit souvent émerger des nouvelles questions et des nouveaux objets de questionnement, étrangers aux réflexions des autres branches médicales - devient un lieu de production et de circulation intense des savoirs sur cet état féminin. Une question surgit à ce moment, dans le cadre de la réflexion des médecins légistes sur le crime d'infanticide: est-il possible qu'une femme ait pu ignorer sa propre grossesse? L'article explore comment la question se pose au carrefour de plusieurs disciplines, dont l'obstétrique, la médecine légale et la psychiatrie. Cependant un point obscur demeure : cela doit-il être considéré d'un point de vue médical ou juridique?

At the beginning of the nineteenth century, pregnancy was one of the privileged objects of attention for French medicine, especially forensics. From this moment on, the texts of this new discipline represented a place of production and circulation of knowledge about this feminine condition, sometimes anticipating issues which had not yet been considered by other medical branches. A specific question arose at this time, in the context of forensic discussions about the crime of infanticide: was it possible for a woman not to be aware of her own pregnancy? This article explores how this question came across different disciplines, such as obstetrics, forensics and psychiatry. However, during the whole $19^{\text {th }}$ century, it was still not very clear whether the issue should be tackled from a medical or a legal point of view.

An der Wende zum 19. Jahrhundert waren Schwangerschaften in Frankreich ein bevorzugtes Reflexionsobjekt der Gerichtsmedizin. Ab dieser Zeit wurde diese neue Disziplin - in der oft neue 
Fragen und Untersuchungsobjekte auftauchten, die in anderen Teilen der Medizin nicht reflektiert wurden - zu einem Ort der intensiven Produktion und Zirkulation von Wissen über diesen weiblichen Zustand. Eine Frage tauchte dabei im Zusammenhang mit der Diskussion über forensische Verbrechen der Kindestötung auf: ist es möglich, dass eine Frau ihre eigene Schwangerschaft ignorieren kann? Der Artikel untersucht, wie sich die Frage an der Schnittstelle mehrerer Disziplinen wie Geburtshilfe, Gerichtsmedizin und Psychiatrie verortete. Ein dunkler Punkt bleibt jedoch: Ist diese Frage unter medizinischer oder unter rechtlicher Sicht zu behandeln?

\section{AUTEUR}

\section{SILVIA CHILETTI}

Silvia Chiletti est chercheuse post-doctorante au Centre Alexandre-Koyré, UMR 8560 CNRSEHESS-MNHN 